\title{
Determination of total mechanical energy of the universe within the framework of Newtonian mechanics
}

\author{
Dimitar Valev \\ Stara Zagora Department, Solar-Terrestrial Influences Laboratory, \\ Bulgarian Academy of Sciences, 6000 Stara Zagora, Bulgaria
}

October 29, 2018

\begin{abstract}
The recent astronomical observations indicate that the expanding universe having a finite particle horizon is homogeneous, isotropic and asymptotically flat. The Euclidean geometry of the universe enables to determine the total kinetic and gravitational energies of the universe within the framework of the Newtonian mechanics. It has been shown that almost the entire kinetic energy of the universe ensues from the cosmological expansion. Both, the total kinetic and gravitational energies of the universe have been determined in relation to an observer at arbitrary location. It is amazing that the modulus of the total gravitational energy differs from the total kinetic energy with a multiplier close to a unit. Thus, the total mechanical energy of the universe has been found close to zero. Both, the total kinetic energy and the modulus of total gravitational energy of the universe are estimated to $3 / 10$ of its total rest energy $M c^{2}$.
\end{abstract}

Key words: energy of the universe; flat universe; Newtonian mechanics

\section{Introduction}

The problem for the average density of the universe $\bar{\rho}$ acquires significance when it has been shown that the General Relativity allows to reveal the geometry and evolution of the universe by simple cosmological models [1, 2, 3]. Crucial for the universe appears dimensionless total density $\Omega=$ $\frac{\bar{\rho}}{\rho_{c}}$, where $\rho_{c}$ is the critical density of the universe. In the case of $\Omega<1$ (open universe) the global spatial curvature is negative and the geometry of the universe is hyperbolic and in the case of $\Omega>1$ (closed universe) the curvature is positive and the geometry is spherical. In the special case of 
$\Omega=1$ (flat universe) the curvature is zero and the geometry is Euclidean. Until recently scarce information has been available about the density and geometry of the universe. The most trustworthy total matter density $\Omega$ has been determined by measurements of the dependence of the anisotropy of the Cosmic Microwave Background $(C M B)$ upon the angular scale. The recent results show that $\Omega \approx 1 \mp \Delta \Omega$, where the error $\Delta \Omega$ decreases from 0.10 [4, 5] to 0.02 [6], i.e. the density of the universe is close to the critical one and the universe is asymptotically flat (Euclidean). The fact that $\Omega$ is so close to a unit is not accidental since only at $\Omega=1$ the geometry of the universe is flat and the flat universe was predicted from the inflationary theory [7. The total density $\Omega$ includes densities of baryon matter $\Omega_{b} \approx 0.05$, cold dark matter $\Omega_{c} \approx 0.25\left[8\right.$ and dark energy $\Omega_{\Lambda} \approx 0.70$ producing an accelerating expansion of the universe [9, 10].

The found negligible $C M B$ anisotropy $\frac{\delta T}{T} \sim 10^{-5}$ indicates that the early universe was very homogeneous and isotropic [11. Three-dimensional maps of the distribution of galaxies corroborate homogeneous and isotropic universe on large scales greater than 100 Mps [12, 13].

Usually, Einstein pseudotensor is used for determination of the total energy of the universe [14, 15]. This approach is general for open, close and flat anisotropic models, but pseudotensorial calculations are dangerous as they are very coordinate dependent and thus, they may lead to ambiguous results [16]. Euclidean geometry of the universe still enables unambiguous determining of the total kinetic and gravitational energies of the universe, within the framework of classical mechanics.

\section{Determination of the total gravitational energy of the universe}

The finite time of cosmological expansion (age of the universe) and the finite speed of light $c$ set a finite particle horizon beyond which no material signals reach the observer. The finite particle horizon ("radius") of the universe is equal to Hubble distance $R \sim \mathrm{cH}^{-1}$, where $H=H_{0} h \approx 70 \mathrm{kms}^{-1} \mathrm{Mps}^{-1}$ [17] is the Hubble expansion rate and $H^{-1} \approx 14$ Gyr is the Hubble time (age of the universe). Therefore, for an observer in arbitrary location, the universe appears a three-dimensional, homogeneous and isotropic sphere having finite radius (particle horizon) $R \sim c H^{-1}$. The possible matter beyond the particle horizon does not affect the observer.

The gravitational potential energy $U$ of a homogeneous sphere with density $\bar{\rho}$ is:

$$
U=-G \int_{0}^{R} \frac{M(r) d m}{r}=-\frac{16}{3} G \pi^{2} \bar{\rho}^{2} \int_{0}^{R} r^{4} d r=-\frac{3}{5} \frac{G M^{2}}{R}
$$

where $G$ is the gravitational constant, $M(r)=\frac{4}{3} \pi r^{3} \bar{\rho}$ is the mass of a 
sphere with radius $r$ and density $\bar{\rho}, M$ is the mass and $R$ is the radius of the universe.

In consideration of $R \sim c H^{-1}$ and $\bar{\rho}=\Omega \rho_{c}$, we obtain for the mass of the universe:

$$
M=\frac{4}{3} \pi R^{3} \bar{\rho}=\frac{4}{3} \frac{\pi c^{3} \Omega \rho_{c}}{H^{3}}
$$

The critical density of the universe [18] is determined from equation (3):

$$
\rho_{c}=\frac{3 H^{2}}{8 \pi G}
$$

Replacing (3) in (2) we have found equation (4) for the mass of the universe including dark matter and dark energy:

$$
M=\frac{c^{3} \Omega}{2 G H}
$$

Finally, replacing (4) in (11) and on account of $R \sim \mathrm{cH}^{-1}$ we have found for the total gravitational energy of the universe:

$$
U=-\frac{3}{20} \frac{c^{5}}{G H} \Omega^{2}
$$

Similar approach has been used for calculation of the total gravitational energy of a body arising from gravitational interaction of the body with all masses of the observable universe [19, 20].

\section{Determination of the total kinetic and total me- chanical energies of the universe}

The estimation of the total kinetic energy of the universe $T$ is complicated as a result of the diversity of movements of masses in the universe. We suggest that almost all kinetic energy of the universe is a result of the cosmological expansion since it includes movement of the enormous masses (galaxies and clusters of galaxies) with average speed of the order of magnitude of $\frac{c}{2}$. The rotation curves of galaxies show that the majority of stars move into the galaxies with speed less than $v_{0}=3 \times 10^{5} \mathrm{~m} / \mathrm{s}$ [21]. Besides, on rare occasions, the peculiar (non-cosmological) velocities of galaxies exceed this value [22]. On the other hand, the speed of medium-distanced galaxies (and their stars), as a result of the cosmological expansion is of the order of magnitude of $\frac{c}{2}=1.5 \times 10^{8} \mathrm{~m} / \mathrm{s}$. Obviously, the kinetic energy of an "average star" in the universe, ensuing from its peculiar movement, constitutes less than $\left(\frac{v_{0}}{c / 2}\right)^{2} \sim 4 \times 10^{-6}$ part of its kinetic energy, ensuing from the cosmological expansion, therefore, former should be ignored. 
Let us estimate the total kinetic energy of the universe in relation to an observer at arbitrary location. The total kinetic energy of the universe is the sum of the kinetic energy of all masses $m_{i}$ moving in relation to the observer with speed $v_{i}$ determined from Hubble law $v_{i}=H r_{i}$, where $r_{i} \leq c H^{-1}$ is the distance between the observer and mass $m_{i}$ placed within the particle horizon:

$$
T=\frac{1}{2} \sum_{i} m_{i} v_{i}^{2}
$$

Although distant galaxies escape from the observer with speeds $v_{i}$ comparable to $c$, the Newtonian formula (6) of kinetic energy is used. Thus, all calculations are made within the framework of the Newtonian mechanics.

Since for an arbitrary observer the universe appears a three-dimensional, homogeneous and isotropic sphere having finite radius (particle horizon) $R \sim c H^{-1}$, the sum (6) can be replaced by integral (7):

$$
T=\frac{1}{2} \int m v^{2} d r=\frac{1}{2} \int_{0}^{R} 4 \pi r^{2} \rho v_{r}^{2} d r=2 \pi \bar{\rho} \int_{0}^{R} v_{r}^{2} r^{2} d r
$$

Replacing $v_{r}$ with expression from Hubble law $v_{r}=H r$, equation (77) is transformed into (8):

$$
T=2 \pi \bar{\rho} H^{2} \int_{0}^{R} r^{4} d r=\frac{2}{5} \pi \bar{\rho} H^{2} R^{5}
$$

In consideration of $R \sim c H^{-1}$ we obtain:

$$
T=\frac{2}{5} \frac{\pi \bar{\rho} c^{5}}{H^{3}}
$$

Since $\bar{\rho}=\Omega \rho_{c}$ (9) is transformed into (10):

$$
T=\frac{2}{5} \frac{\pi \Omega \rho_{c} c^{5}}{H^{3}}
$$

After replacing (3) in (10) we have found the equation for the total kinetic energy of the universe:

$$
T=\frac{3}{20} \frac{c^{5}}{G H} \Omega
$$

It is amazing that the equations for the total gravitational and kinetic energies of the universe (5) and (11) differ one from another by a multiplier $\Omega \approx 1$.

Clearly, the total mechanical energy of the universe $E$ is:

$$
E=T+U=\frac{3}{20} \frac{c^{5}}{G H} \Omega(1-\Omega)
$$


Since the resent $C M B$ measurements obtain $\Omega \approx 1$, then $E=T+U \approx$ 0 , i.e. the total mechanical energy of the universe is close to zero. This substantial result confirms the conjecture that the gravitational energy of the universe is approximately balanced with its kinetic energy of the expansion [23.

Formulae (5) and (11) show that the total kinetic energy of the universe is close to the modulus of its total gravitational energy:

$$
T \approx|U| \approx \frac{3}{20} \frac{c^{5}}{G H}
$$

From Einstein equation and (4) we can determine the total rest energy of the universe:

$$
E_{0}=M c^{2}=\frac{1}{2} \frac{c^{5}}{G H} \Omega \approx \frac{1}{2} \frac{c^{5}}{G H}
$$

Therefore, the total kinetic energy of the universe is $\frac{3}{10}$ of its total rest energy $E_{0}$. The same is valid for the modulus of the total gravitational energy of the universe, too.

\section{Discussions}

Formulae (13) and (14) show that the total gravitational energy of the universe is $U=-\frac{3}{10} E_{0}=-\frac{3}{10} M c^{2}$, i. e. $30 \%$ of the total rest energy of the universe. On the other hand, the gravitational energy of a sphere having mass $m$ and radius $r$ equal to its Schwarzschild's radius $r_{s}=\frac{2 G m}{c^{2}}$ is:

$$
U=-\frac{3}{5} \frac{G m^{2}}{r_{s}}=-\frac{3}{10} m c^{2}
$$

which also appears $30 \%$ of the total rest energy of the mass $m$.

This suggests that the particle horizon ("radius") of the universe $(R)$ coincides with Schwarzschild's radius of the universe $\left(R_{s}\right)$. It is easy to prove this statement using the following:

On account of $\Omega=\frac{\bar{\rho}}{\rho_{c}} \approx 1$ and $H \sim c R^{-1}$ we obtain:

$$
\Omega=\frac{\bar{\rho}}{\rho_{c}}=\frac{3 M / 4 \pi R^{3}}{3 H^{2} / 8 \pi G}=\frac{2 G M}{R^{3} H^{2}} \sim \frac{2 G M}{R c^{2}} \approx 1
$$

Therefore, $R \sim c H^{-1} \approx \frac{2 G M}{c^{2}} \equiv R_{s}$, i. e. the particle horizon ("radius") of the universe coincides with Schwarzschild's radius of the universe.

It is interesting that formulae for the total mass and rest energy of the universe (44) and (14) would be found by dimensional analysis, without consideration of the total density of the universe. In fact, a quantity with mass dimension $M=k c^{\alpha} G^{\beta} H^{\gamma}$ could be composed by using the fundamental 
parameters - speed of the light $(c)$, gravitational constant $(G)$ and Hubble constant $(H)$. Dimensional analysis gave $\alpha=3$ and $\beta=\gamma=-1$. Therefore:

$$
M=k \frac{c^{3}}{G H}
$$

where $k \sim 1$.

Analogously, a quantity with dimension of energy $E_{0}=k_{0} c^{a} G^{b} H^{d}$ could be composed by parameters $c, G$ and $H$. In this case dimensional analysis has shown that $a=5$ and $b=d=-1$. Therefore,

$$
E_{0}=k_{0} \frac{c^{5}}{G H}
$$

where $k_{0} \sim 1$.

Obviously, the formulae (17) and (18) coincide with formulae of total mass and rest energy of the universe (44) and (14), with accuracy to a factor $k=\frac{1}{2}$. Thus, the total mass and rest energy of the universe have been found by original independent approach, namely dimensional analysis, which reinforces our presumptions for accepted cosmological model.

\section{Conclusions}

The recent astronomical observations indicate that the expanding universe having a finite particle horizon is homogeneous, isotropic and flat. The Euclidean geometry of the universe enables to determine the total kinetic and gravitational energies of the universe within the framework of the Newtonian mechanics.

Based on the simple homogeneous and isotropic model of the flat universe which expands according to Hubble law, we have found equations (5) and (11) for the total gravitational and kinetic energy of the universe, respectively. It is amazing that these equations differ by a multiplier $\Omega \approx 1$. That is, the modulus of the total gravitational energy of the universe is close to its total kinetic energy and therefore the total mechanical energy $E$ of the universe is close to zero, i. e. the gravitational energy of the universe is balanced with its kinetic energy of the expansion.

Both, the total kinetic and gravitational energies of the universe have been determined in relation to an observer at arbitrary location. The total kinetic energy and the modulus of the total gravitational energy of the universe are estimated to $\frac{3}{10}$ of its total rest energy $M c^{2}$.

Finally, it has been shown that the particle horizon ("radius") of the universe coincides with Schwarzschild's radius of the universe. Besides, the total mass and rest energy of the universe have been estimated by dimensional analysis, without consideration of total density of the universe. 


\section{References}

[1] Friedman A., 1922, Z. Physik, 10, 377.

[2] Lemaitre G., 1927, Ann. Soc. Sci. Brux., 47A, 49.

[3] Einstein A., de Sitter W. , 1932, Proc. Nat. Acad. Sci. USA, 18, 213.

[4] de Bernardis P. et al., 2000, Nature, 404, 955.

[5] Balbi A. et al., 2000, Astrophys. J., 545, L1.

[6] Spergel D. N. et al., 2003, Astrophys. J. Suppl. Series, 148, 175.

[7] Guth A. H., 1981, Phys. Rev. D, 23, 347.

[8] Peacock J. A. et al., 2001, Nature, 410, 169.

[9] Riess A. G. et al., 1998, Astron. J., 116, 1009.

[10] Perlmutter S. et al., 1999, Astrophys. J., 517, 565.

[11] Bennett C. L. et al., 1996, Astrophys. J., 464, L1.

[12] Shectman S. A. et al., 1996, Astrophys. J., 470, 172.

[13] Stoughton C. et al., 2002, Astron. J., 123, 485.

[14] Rosen N., 1994, Gen. Relativ. Gravit., 26, 319.

[15] Johri V. et al., 1995, Gen. Relativ. Gravit., 27, 313.

[16] Banerjee N., Sen S.,1997, Pramana - J. Phys. 49, 609.

[17] Mould J. R. et al., 2000, Astrophys. J., 529, 786.

[18] Peebles P. J. E.,1971, Physical Cosmology, Princeton Univ. Press, NJ.

[19] Woodward J. F. et al., 1975, Phys. Rev. D, 11,1371.

[20] Valev D., Compt. rend. Acad. bulg. Sci., Spec. Iss.: Fundamental Space Research, 2009, 258; http://arxiv.org/abs/0902.1412.

[21] Sofue Y., 1996, Astrophys. J., 458, 120.

[22] Strauss M. A., Willick J. A., 1995, Phys. Reports, 261, 271.

[23] Lightman A. P., 1984, Science, 224, 863. 